\title{
COVID-19 vaccines and thrombosis
}

\author{
Giuseppe Famularo ${ }^{1}$
}

Received: 18 July 2021 / Accepted: 29 July 2021 / Published online: 12 October 2021

(c) Società Italiana di Medicina Interna (SIMI) 2021

\section{Dear Editor,}

Carli and colleagues recently described a patient with deep vein thrombosis occurring after the second dose of the messenger RNA Covid-19 vaccine BNT 162b2 (Comirnaty, Pfizer/BioNTech) [1]. I report a case of renal vein thrombosis associated with the BNT $162 \mathrm{~b} 2$ vaccine.

On June 1, 2021 a 71-year-old Caucasian man attended our emergency department with a 4-day history of abdominal pain and fever. He reported no chest pain, shortness of breath, swelling, redness, pallor, or cold in any limb. On April 9 and 30, he had received the two scheduled doses of the messenger RNA Covid-19 vaccine BNT 162b2 (Comirnaty, Pfizer/BioNTech). No symptoms or signs of local or systemic reactogenicity were recognized after both doses. The patient's previous history was remarkable for autoimmune chronic hepatitis and adenocarcinoma of the descending colon for which a colectomy had been performed 2 years prior. There was no personal or family history of venous thromboembolism or other thrombotic disorders, bleeding, autoimmune, or allergic disorders. His medications included azathioprine, prednisone, rifaximin, and ursodeoxycholic acid.

At admission, the patient was febrile $\left(38.2{ }^{\circ} \mathrm{C}\right)$ with normal vital signs and physical examination. The white cell count was $18,550 / \mathrm{mm}^{3}$, with lymphocytes of $1200 / \mathrm{mm}^{3}$, platelets were $276,000 / \mathrm{mmc}$, C-reactive protein (CRP) level was of $16.4 \mathrm{mg} / \mathrm{L}$, bilirubin was $1.5 \mathrm{mg} / \mathrm{dl}$, creatinine $1.7 \mathrm{mg} / \mathrm{dl}$, and D-dimer $2705 \mathrm{ng} / \mathrm{ml}$ (normal 250-500); fibrinogen, the international normalized ratio, and the thromboplastin time were normal. SARS-CoV-2 antigen and RT-PCR were negative on a nasopharyngeal swab. Klebsiella variicola was isolated from a sample of urine, blood

The article belongs to COVID 19.

Giuseppe Famularo

gfamularo@scamilloforlanini.rm.it

1 Internal Medicine, San Camillo Hospital, Circonvallazione Gianicolense, 00152 Rome, Italy cultures grew no pathogens, and procalcitonin was normal. A contrast-enhanced computed tomography (CT) showed splenomegaly (longitudinal diameter $14 \mathrm{~cm}$ ) and thrombosis of the left renal vein. A thrombophilia screen, including resistance to activated protein $\mathrm{C}$, protein $\mathrm{C}$ and $\mathrm{S}$ activity, antiphospholipid antibodies (cardiolipin, $\beta 2$ glycoprotein, and lupus), and homocysteine, was normal. Meropenem and anticoagulant therapy with enoxaparin were started, the patient's condition improved, and he was apyrexial and free of symptoms on the 8th day of hospital stay. Enoxaparin was then switched to dabigatran (110 mg twice per day) and he was discharged on day 9th. At follow-up 1 month later, the patient was doing well, blood levels of CRP and D-dimer were normal, and imaging studies showed complete resolution of the left renal vein thrombosis and no new or progressive thrombosis.

Thrombosis of the left renal vein was diagnosed 4 weeks after this patient was given the second dose of the messenger RNA Covid-19 vaccine BNT 162b2. On consideration of clinical presentation, the timing of onset, the atypical location of thrombosis, and the absence of established risk factors for venous thromboembolism we assumed this unusual thrombotic event could have been linked with the BNT $162 \mathrm{~b} 2$ vaccine. I acknowledge the patient's history of chronic autoimmune hepatitis and colon cancer could be confounding. However, clinical, laboratory, and imaging work-up did not show any evidence of active hepatitis or cancer relapse. Furthermore, renal vein thrombosis has not been consistently reported in patients with infections of the urinary tract. As a matter of fact, this patient had none of the established risk factors for renal vein thrombosis such as nephrotic syndrome, membranous glomerulonephritis, renal cell carcinoma, surgery, urological procedures, kidney transplantation, and abdominal trauma [2]. In addition, autoimmune hepatitis as well as other chronic liver disorders are not associated with renal vein thrombosis.

Case reports and small series have suggested a potential safety signal associated with adenoviral vector Covid-19 vaccines involving venous thrombosis and thrombocytopenia (vaccine-induced immune thrombotic thrombocytopenia, 
VITT [3-5]. Of note, VITT was easily ruled out in this patient since he had normal platelet count at presentation and throughout the subsequent course and there was no evidence of multifocal thrombosis of the splanchnic and cerebral veins. Even though vaccine-induced inflammation and immune activation may underlie a definite thrombotic risk, it remains unclear whether Covid-19 vaccines could cause a clinically relevant prothrombotic state and what vaccinespecific factors could be contributing to. As of today, however, it is clear that Covid-19 vaccines as such could not be recognized as a risk factor for thromboembolic disease. However, this case is of concern and continued monitoring is needed to further assess any possible thrombotic signal.

Funding None.

Data availability All data are fully available.

\section{Declarations}

Conflict of interest The author declares that he has no conflict of interest.

Human and animal rights All procedures performed in studies involving human participants were in accordance with the ethical standards of the institutional and/or national research committee and with the 1964 Helsinki declaration and its later amendments or comparable ethical standards.

Informed consent For this type of study, formal consent is not required.

\section{References}

1. Carli G, Nichele I, Ruggeri M, Barra S, Tosetto A (2021) Deep vein thrombosis (DVT) occurring shortly after the second dose of mRNA SARS-CoV-2 vaccine. Intern Emerg Med 16:803-804

2. Wysokinski WE, EL Gosk-BierskaI G, Grill D, Wiste H, McBane RD (2008) Clinical characteristics and long-term follow-up of patients with renal vein thrombosis. Am J Kidney Dis 51:224-232

3. Greinacher A, Thiele T, Warkentin TE, Weisser K, Kyrle P, Eichinger S (2021) Thrombotic thrombocytopenia after ChAdOx1 vaccination. N Engl J Med 384:2092-2101

4. Schultz NH, Sorvoll IH, Michelsen AE et al (2021) Thrombosis and thrombocytopenia after ChAdOx $1 \mathrm{nCoV}-19$ vaccination. $\mathrm{N}$ Engl J Med 384:2124-2130

5. Muir K-L, Kallam A, Koepsell SA, Gundabolu K (2021) Thrombotic thrombocytopenia after Ad26.COV2.S vaccination. N Eng1 J Med 384:1964-1965

Publisher's Note Springer Nature remains neutral with regard to jurisdictional claims in published maps and institutional affiliations. 